\title{
Generalized Gronwall fractional summation inequalities and their applications
}

Run $\mathrm{Xu}^{1 *}$ and Ying Zhang ${ }^{2}$
*Correspondence: xurun2005@163.com

${ }^{1}$ Department of Mathematics, Qufu Normal University, Qufu, Shandong 273165, People's Republic of China Full list of author information is

available at the end of the article
MSC: 39A12; 26A33

Keywords: Gronwall's inequality; fractional difference; fractional sum; fractional difference equation; fractional sum equation

\section{Introduction}

In recent years, the fractional differential and fractional integrals have been adopted in various fields of science and engineering, which can be used to describe certain phenomena, reflect some physiochemical properties, and provide accurate models for the systems under consideration. Some applications of fractional calculus include fluid flow, rheology, dynamical processes in self-similar and porous structures, electrical networks, probability and statistics, control theory of dynamical systems, chemical physics, optics, and signal processing, economics, and so on. Therefore, they are receiving extensive attention from a variety of domains. References $[1,2]$ introduced the definitions of fractional calculus, theorems and basic analytic solutions of the fractional equation in detail. At the same time, the fractional difference equations, fractional sum equations, and fractional inequalities also play important roles in many areas. In 1989, Miller and Ross [3] defined a fractional sum of order $\alpha>0$ via the solution of a linear difference equation and proved some basic properties of this operator. After that, many authors followed up in various directions [4-17].

In 2000, Hirota [18] defined the fractional order difference operator $\nabla^{\alpha}$ where $\alpha$ was a real number, using Taylor's series. In 2003, Nagai [19] adopted another definition for the fractional difference by modifying Hirota's definition. The definition in [19] contained the $\nabla$ operator and the term $(-1)^{j}$ inside the summation index. Therefore this definition was difficult use in studying the properties of solutions of fractional difference equations. To avoid this, Deekshitulu and Mohan [20] modified the definition and rearranged the terms in the definition of Nagai [19]. Then they defined the fractional sum operator $\nabla^{-\alpha}$ and the fractional difference operator $\nabla^{\alpha}$. Recently, they discussed some basic inequalities, 
comparison theorems, and qualitative properties of the solutions of fractional difference equations in [21-24]. Under the definition in [20], they also considered an initial value problem of fractional order and obtained some useful fractional difference inequalities of Gronwall-Bellman type [25].

In 2009, Atici and Eloe [26] introduced the definition of the fractional sum and the fractional difference: $\nabla^{-\alpha}, \nabla^{\alpha}, \Delta^{-\alpha}, \Delta^{\alpha}$. In 2012, based on the definition, Atici and Eloe [27] and Ferreira [28] studied some discrete fractional Gronwall's inequalities as regards $\nabla^{-\alpha}, \Delta^{-\alpha}$ separately. They all adopted the same method: After establishing a comparison theorem, they gave an explicit solution to the linear discrete fractional sum equation of the initial value problem, which allowed them to state and prove an analog of Gronwall's inequality on discrete fractional calculus.

In 2011, Cheng [29] presented another form of definition. He provided some basic properties of fractional difference and summation, and established the theory symmetrical to the fractional differential equation.

In this paper, we will introduce some inequalities and their applications based on the definition in [29] to demonstrate the qualitative properties of solutions to some fractional summation equations. The proof is based on the iterative method.

Definition 1.1 [29] Let $v$ be nonnegative real number, define the $v$-order summation of $x(n)$ as

$$
\nabla^{-v} x(n)=\left[\begin{array}{l}
v \\
n
\end{array}\right] * x(n)=\sum_{r=0}^{n}\left[\begin{array}{c}
v \\
n-r
\end{array}\right] x(r),
$$

where $\left[\begin{array}{l}v \\ n\end{array}\right]=\frac{v(v+1) \cdots(v+n-1)}{n !}, *$ is the convolution operator.

Definition 1.2 [29] Let $\mu$ be positive real number and $m$ be the minimum positive integer which is greater than $\mu(m-1 \leq \mu \leq m)$. Define the $\mu$-order difference of $x(n)$ as

$$
\nabla^{\mu} x(n)=\nabla^{m} \nabla^{-(m-\mu)} x(n)
$$

where $\nabla^{m}$ is the $m$ th-order backward difference operator.

Definition 1.3 [29] Define the discrete Mittag-Leffler function $F_{\alpha, \beta}(\lambda, n)$ as

$$
\begin{aligned}
& F_{\alpha, \beta}(\lambda, n)=\sum_{k=0}^{\infty} \lambda^{k}\left[\begin{array}{c}
\alpha k+\beta \\
n
\end{array}\right] \quad(|\lambda|<1), \\
& F_{\alpha}(\lambda, n)=F_{\alpha, 1}(\lambda, n)=\sum_{k=0}^{\infty} \lambda^{n}\left[\begin{array}{c}
\alpha k+1 \\
n
\end{array}\right] \quad(|\lambda|<1) .
\end{aligned}
$$

In [11], Cheng gave the discrete fractional summation Gronwall inequality.

Theorem 1.1 [29] Suppose that $\beta>0$. Let $u_{n}, a_{n}$, and $g_{n}$ be nonnegative functions, where $g_{n}$ is also monotone and nondecreasing and satisfies $0 \leq g_{n} \leq M(0 \leq M<1), 0 \leq n \leq N$. If

$$
u_{n} \leq a_{n}+g_{n} \nabla^{-\beta} u_{n}
$$


then

$$
u_{n} \leq a_{n}+\sum_{k=1}^{\infty}\left(g_{n}\right)^{k} \nabla^{-k \beta} a_{n}
$$

In [30], Zhang et al. have extended the result and given the following conclusion.

Theorem 1.2 [30] Let $\beta>0, p \geq r>0, u_{n}, a_{n}, g_{n}$ be nonnegative functions, where $g_{n}$ is also monotone and nondecreasing and satisfies $0 \leq g_{n} \leq M(0 \leq M<1), 0 \leq n \leq N$. If

$$
\left(u_{n}\right)^{p} \leq a_{n}+g_{n} \nabla^{-\beta}\left(u_{n}\right)^{r},
$$

then

$$
u_{n} \leq\left\{a_{n}+D_{n}+\sum_{k=1}^{\infty}\left(C g_{n}\right)^{k} \nabla^{-k \beta} D_{n}\right\}^{\frac{1}{p}},
$$

where

$$
\begin{aligned}
& D_{n}=g_{n} \nabla^{-\beta}\left\{\frac{r}{p} k^{\frac{r-p}{p}} a_{n}+\frac{p-r}{p} k^{\frac{r}{p}}\right\}, \\
& C=\frac{r}{p} k^{\frac{r-p}{p}}, \quad k>1 .
\end{aligned}
$$

In order to prove our results, we need the following basic information.

Lemma 1.1 [29] Let $\mu>0, v>0$, then $\nabla^{-\mu} \nabla^{-v} x(n)=\nabla^{-(u+v)} x(n)$.

Lemma 1.2 [29] $\nabla^{-\beta}\left[\begin{array}{c}k \\ n\end{array}\right]=\left[\begin{array}{c}k+\beta \\ n\end{array}\right], k, n, \beta>0$.

\section{Main results}

Theorem 2.1 Suppose that $\beta>0, u_{n}, a_{n}, g_{n}, h_{n}$ are nonnegative functions, $g_{n}$ and $h_{n}$ are also monotone and nondecreasing, $u_{n} \leq c, g_{n} \leq M_{1}, h_{n} \leq M_{2}\left(M_{1} M_{2}<1,0 \leq n \leq N\right)$. If

$$
u_{n} \leq a_{n}+g_{n} \nabla^{-\beta} h_{n} u_{n}
$$

then

$$
u_{n} \leq a_{n}+\sum_{k=1}^{\infty} g_{n}^{k} h_{n}^{k} \nabla^{-k \beta} a_{n}
$$

Proof Let $B u_{n}=g_{n} \nabla^{-\beta} h_{n} u_{n}$. We find that $B$ is linear and nondecreasing under the conditions that $u_{n}, g_{n}, h_{n}$ are nonnegative functions and $g_{n}, h_{n}$ are also nondecreasing. Therefore, (2.1) turns into

$$
u_{n} \leq a_{n}+B u_{n} .
$$


Then we have

$$
\begin{aligned}
u_{n} & \leq a_{n}+B\left(a_{n}+B u_{n}\right)=a_{n}+B a_{n}+B^{2} u_{n} \leq \cdots \\
& \leq a_{n}+\sum_{k=1}^{m-1} B^{k} a_{n}+B^{m} u_{n}
\end{aligned}
$$

and

$$
\begin{aligned}
& B u_{n}=g_{n} \nabla^{-\beta} h_{n} u_{n} \leq c M_{1} M_{2}\left[\begin{array}{c}
1+\beta \\
n
\end{array}\right], \\
& B^{2} u_{n}=g_{n} \nabla^{-\beta} h_{n}\left(g_{n} \nabla^{-\beta} h_{n} u_{n}\right) \leq c\left(M_{1} M_{2}\right)^{2}\left[\begin{array}{c}
1+2 \beta \\
n
\end{array}\right], \\
& \vdots \\
& B^{m} u_{n}=g_{n}^{m} h_{n}^{m} \nabla^{-m \beta} u_{n} \leq c\left(M_{1} M_{2}\right)^{m}\left[\begin{array}{c}
1+m \beta \\
n
\end{array}\right] .
\end{aligned}
$$

Noticing that $\left|M_{1} M_{2}\right|<1$, then

$$
\sum_{m=1}^{\infty}\left(M_{1} M_{2}\right)^{m}\left[\begin{array}{c}
1+m \beta \\
n
\end{array}\right]=F_{\beta, 1}\left(M_{1} M_{2}, n\right)-1
$$

Hence we have

$$
\lim _{m \rightarrow \infty}\left(M_{1} M_{2}\right)^{m}\left[\begin{array}{c}
1+m \beta \\
n
\end{array}\right]=0 .
$$

From (2.4) we get

$$
\lim _{m \rightarrow \infty} B^{m} u_{n} \leq 0 .
$$

Due to the fact that $B^{m} u_{n} \geq 0$, we have

$$
\lim _{m \rightarrow \infty} B^{m} u_{n}=0 .
$$

Taking the limit as $m \rightarrow \infty$ on both sides of (2.3) we get

$$
u_{n} \leq a_{n}+\sum_{k=1}^{\infty} B^{k} a_{n} \leq a_{n}+\sum_{k=0}^{\infty} g_{n}^{k} h_{n}^{k} \nabla^{-k \beta} a_{n}
$$

This completes the proof of Theorem 2.1.

Remark 2.1 If $h_{n} \equiv 1,(2.2)$ becomes Theorem 2.2 of Chapter 3 in [29]:

$$
u_{n} \leq a_{n}+\sum_{k=1}^{\infty} g_{n}^{k} \nabla^{-k \beta} a_{n}
$$


Corollary 2.1 With the conditions of Theorem 2.1, let $g_{n}=a, 0<a M_{2}<1$. If

$$
u_{n} \leq a_{n}+a \nabla^{-\beta} h_{n} u_{n}
$$

then

$$
u_{n} \leq a_{n}+\sum_{k=1}^{\infty} a^{k} h_{n}^{k} \nabla^{-k \beta} a_{n}
$$

Corollary 2.2 Suppose that the conditions of Corollary 2.1 hold, $a_{n}$ is monotone increasing and $0<a M_{2}<1$. If

$$
u_{n} \leq a_{n}+a \nabla^{-\beta} h_{n} u_{n}
$$

then

$$
u_{n} \leq a_{n} F_{\beta, 1}\left(a M_{2}, n\right) .
$$

Proof Under the conditions of Corollary 2.1 and $a_{n}$ is monotone increasing, and we get

$$
\begin{aligned}
u_{n} & \leq a_{n}+\sum_{k=1}^{\infty} a^{k} h_{n}^{k} \nabla^{-k \beta} a_{n} \leq a_{n}+a_{n} \sum_{k=1}^{\infty} a^{k} h_{n}^{k} \nabla^{-k \beta}\left[\begin{array}{l}
1 \\
n
\end{array}\right] \\
& \leq a_{n} \sum_{k=0}^{\infty}\left(a M_{2}\right)^{k}\left[\begin{array}{c}
k \beta+1 \\
n
\end{array}\right]=a_{n} F_{\beta, 1}\left(a M_{2}, n\right) \quad\left(0<a M_{2}<1\right) .
\end{aligned}
$$

Corollary 2.3 Suppose that the conditions of Theorem 2.1 hold, if $a_{n}$ is monotone increasing, then from

$$
u_{n} \leq a_{n}+g_{n} \nabla^{-\beta} h_{n} u_{n}
$$

we get

$$
u_{n} \leq a_{n} \sum_{k=0}^{\infty}\left(M_{1} M_{2}\right)^{k} \nabla^{-k \beta}\left[\begin{array}{l}
1 \\
n
\end{array}\right]=a_{n} F_{\beta, 1}\left(M_{1} M_{2}, n\right) .
$$

Theorem 2.2 Suppose that $\beta>0, u_{n}$, and $a_{n}$ are nonnegative functions, $\varphi(t)$ is monotone and nondecreasing, $\varphi(t+s) \leq \varphi(t)+\varphi(s), \varphi(t) \leq L t, 0<L<1$. If

$$
u_{n} \leq a_{n}+\nabla^{-\beta} \varphi\left(u_{n}\right)
$$

then

$$
u_{n} \leq \sum_{k=0}^{\infty} \nabla^{-k \beta} \varphi^{k}\left(a_{n}\right)
$$


Proof Let $B u_{n}=\nabla^{-\beta} \varphi\left(u_{n}\right)$. Under the conditions that $\varphi(a+b) \leq \varphi(a)+\varphi(b)$ and $\varphi(t)$ is monotone and nondecreasing, we have

$$
\begin{aligned}
& \varphi\left(\nabla^{-\beta} \varphi\left(u_{n}\right)\right)=\varphi\left(\sum_{r=0}^{n}\left[\begin{array}{c}
v \\
n-r
\end{array}\right] \varphi(u(r))\right) \leq \sum_{r=0}^{n}\left[\begin{array}{c}
v \\
n-r
\end{array}\right] \varphi^{2}(u(r))=\nabla^{-\beta} \varphi^{2}\left(u_{n}\right), \\
& \varphi^{2}\left(\nabla^{-\beta} \varphi\left(u_{n}\right)\right) \leq \nabla^{-\beta} \varphi^{3}\left(u_{n}\right),
\end{aligned}
$$

then for all $n \in N_{0}^{+}$,

$$
\begin{aligned}
u_{n} \leq & a_{n}+B u_{n}=a_{n}+\nabla^{-\beta} \varphi\left(u_{n}\right) \\
\leq & a_{n}+\nabla^{-\beta} \varphi\left(a_{n}+B u_{n}\right)=a_{n}+\nabla^{-\beta} \varphi\left(a_{n}+\nabla^{-\beta} \varphi\left(u_{n}\right)\right) \\
\leq & a_{n}+\nabla^{-\beta} \varphi\left(a_{n}\right)+\nabla^{-\beta} \varphi\left(\nabla^{-\beta} \varphi\left(u_{n}\right)\right) \\
\leq & a_{n}+\nabla^{-\beta} \varphi\left(a_{n}\right)+\nabla^{-2 \beta} \varphi^{2}\left(u_{n}\right) \\
\leq & a_{n}+\nabla^{-\beta} \varphi\left(a_{n}\right)+\nabla^{-2 \beta} \varphi^{2}\left(a_{n}+\nabla^{-\beta} \varphi\left(u_{n}\right)\right) \\
\leq & a_{n}+\nabla^{-\beta} \varphi\left(a_{n}\right)+\nabla^{-2 \beta} \varphi^{2}\left(a_{n}\right)+\nabla^{-3 \beta} \varphi^{3}\left(u_{n}\right) \\
\leq & \cdots \\
\leq & a_{n}+\nabla^{-\beta} \varphi\left(a_{n}\right)+\nabla^{-2 \beta} \varphi^{2}\left(a_{n}\right)+\nabla^{-3 \beta} \varphi^{3}\left(a_{n}\right)+\cdots \\
& +\nabla^{-(m-1) \beta} \varphi^{m-1}\left(a_{n}\right)+\nabla^{-m \beta} \varphi^{m}\left(u_{n}\right),
\end{aligned}
$$

that is,

$$
u_{n} \leq \sum_{k=0}^{m-1} \nabla^{-k \beta} \varphi^{k}\left(a_{n}\right)+\nabla^{-m \beta} \varphi^{m}\left(u_{n}\right)
$$

Noticing that $\varphi\left(u_{n}\right) \leq L u_{n},|L|<1$, and $u_{n}$ is bounded for $0 \leq n \leq N$, suppose that $0<u_{n} \leq C$, then we get

$$
\begin{aligned}
& \nabla^{-\beta} \varphi\left(u_{n}\right) \leq L \nabla^{-\beta} u_{n} \leq L \nabla^{-\beta} C=C L\left[\begin{array}{c}
1+\beta \\
n
\end{array}\right] \\
& \nabla^{-2 \beta} \varphi^{2}\left(u_{n}\right) \leq C L^{2}\left[\begin{array}{c}
1+2 \beta \\
n
\end{array}\right] \\
& \nabla^{-3 \beta} \varphi^{3}\left(u_{n}\right) \leq C L^{3}\left[\begin{array}{c}
1+3 \beta \\
n
\end{array}\right] \\
& \vdots \\
& \nabla^{-m \beta} \varphi^{m}\left(u_{n}\right) \leq C L^{m}\left[\begin{array}{c}
1+m \beta \\
n
\end{array}\right] .
\end{aligned}
$$


From the fact that the series

$$
\sum_{m=0}^{\infty} L^{m}\left[\begin{array}{c}
1+m \beta \\
n
\end{array}\right]
$$

converges to $F_{\beta, 1}(L, n),|L|<1$, we have

$$
\lim _{m \rightarrow \infty} L^{m}\left[\begin{array}{c}
1+m \beta \\
n
\end{array}\right]=0 .
$$

Due to the fact that $\nabla^{-m \beta} \varphi^{m}\left(u_{n}\right) \geq 0$, from (2.8) and (2.9) we have

$$
\lim _{m \rightarrow \infty} \nabla^{-m \beta} \varphi^{m}\left(u_{n}\right)=0
$$

taking the limit as $m \rightarrow \infty$ on both sides of (2.7), we have

$$
u_{n} \leq \sum_{k=0}^{\infty} \nabla^{-k \beta} \varphi^{k}\left(a_{n}\right)
$$

This completes the proof of Theorem 2.2.

By choosing some particular functions $\varphi(t)$, we can get the corresponding results. For example, suppose that $a_{n}, u_{n}, \beta$ are the same as in Theorem 2.2. Let $\varphi(t)=\frac{1}{2} \sin t\left(0 \leq t \leq \frac{\pi}{2}\right)$, $0 \leq u_{n} \leq \frac{\pi}{2}$, then $\varphi(t)$ satisfies $\varphi(t+s) \leq \varphi(t)+\varphi(s), \varphi(t) \leq \frac{1}{2} t$. If

$$
u_{n} \leq a_{n}+\frac{1}{2} \nabla^{-\beta} \sin u_{n}
$$

then

$$
u_{n} \leq \sum_{k=0}^{\infty}\left(\frac{1}{2}\right)^{k} \nabla^{-k \beta} \sin ^{k} a_{n} .
$$

Furthermore, if $a_{n} \equiv 1$, then from $\sin t \leq t$, we have

$$
u_{n} \leq \sum_{k=0}^{\infty}\left(\frac{1}{2}\right)^{k} \nabla^{-k \beta} 1 \leq \sum_{k=0}^{\infty}\left(\frac{1}{2}\right)^{k}\left[\begin{array}{c}
1+k \beta \\
n
\end{array}\right]
$$

\section{Applications}

Consider the fractional difference equation

$$
\left\{\begin{array}{l}
\nabla^{\alpha} y(n)=f(n, y(n)) \\
\left.\nabla^{\alpha-1} y(n)\right|_{n=-1}=\eta
\end{array}\right.
$$

where $0<\alpha<1,0 \leq n \leq N<\infty$.

From Proposition 3.1 in Chapter 3 of [29], we know that the problem (3.1) is equivalent to the summation equation

$$
y(n)=\eta\left[\begin{array}{l}
\alpha \\
n
\end{array}\right]+\nabla^{-\alpha} f(n, y(n))
$$


Suppose that $z_{n}$ is the solution of the initial value problem

$$
\left\{\begin{array}{l}
\nabla^{\alpha} z(n)=f(n, z(n)), \\
\left.\nabla^{\alpha-1} z(n)\right|_{n=-1}=\tilde{\eta},
\end{array}\right.
$$

$0<\alpha<1,0 \leq n \leq N<\infty$, then (3.3) is equivalent to the summation equation

$$
z(n)=\widetilde{\eta}\left[\begin{array}{l}
\alpha \\
n
\end{array}\right]+\nabla^{-\alpha} f(n, z(n)) .
$$

Theorem 3.1 Suppose that $f(n, t)$ satisfies the condition

$$
|f(n, z)-f(n, y)| \leq h_{n}|z-y|
$$

where $h_{n}$ is a monotone nondecreasing positive function, $\left|h_{n}\right|<1$. Then the solutions of (3.1) rely on the initial value continuously.

Proof From (3.2), (3.4), and (3.5) we have

$$
\begin{aligned}
|z(n)-y(n)| & \leq|\widetilde{\eta}-\eta|\left[\begin{array}{l}
\alpha \\
n
\end{array}\right]+\nabla^{-\alpha}|f(n, z(n))-f(n, y(n))| \\
& \leq|\tilde{\eta}-\eta|\left[\begin{array}{l}
\alpha \\
n
\end{array}\right]+\nabla^{-\alpha} h_{n}|z(n)-y(n)| .
\end{aligned}
$$

From Corollary 2.1, we have

$$
\begin{aligned}
|z(n)-y(n)| & \leq|\tilde{\eta}-\eta|\left[\begin{array}{c}
\alpha \\
n
\end{array}\right]+\sum_{k=1}^{\infty} h_{n}^{k} \nabla^{-k \alpha}|\tilde{\eta}-\eta|\left[\begin{array}{l}
\alpha \\
n
\end{array}\right] \\
& =|\tilde{\eta}-\eta| \sum_{k=0}^{\infty} h_{n}^{k}\left[\begin{array}{c}
k \alpha+\alpha \\
n
\end{array}\right]=|\tilde{\eta}-\eta| F_{\alpha, \alpha}\left(h_{n}, n\right), \quad\left|h_{n}\right|<1 .
\end{aligned}
$$

Then the solutions of (3.1) rely on the initial value continuously.

Remark 3.1 The condition (3.5) generalizes the Lipschitz condition, then Theorem 3.1 is a promotion of Theorem 3.2 in Chapter 3 of [29].

Theorem 3.2 Suppose that $f(n, t)$ satisfies the condition

$$
|f(n, z)-f(n, y)| \leq \varphi(|z-y|)
$$

where $\varphi(t)$ is the same as in Theorem 2.2. Then the solutions of (3.1) rely on the initial value continuously. 
Proof From (3.2), (3.4), and (3.6) we have

$$
\begin{aligned}
|z(n)-y(n)| & \leq|\widetilde{\eta}-\eta|\left[\begin{array}{l}
\alpha \\
n
\end{array}\right]+\nabla^{-\alpha}|f(n, z(n))-f(n, y(n))| \\
& \leq|\widetilde{\eta}-\eta|\left[\begin{array}{l}
\alpha \\
n
\end{array}\right]+\nabla^{-\alpha} \varphi(|z(n)-y(n)|) .
\end{aligned}
$$

By Theorem 2.2 we get

$$
\begin{aligned}
|z(n)-y(n)| & \leq \sum_{k=0}^{\infty} \nabla^{-k \alpha} \varphi^{k}\left(|\widetilde{\eta}-\eta|\left[\begin{array}{l}
\alpha \\
n
\end{array}\right]\right) \\
& \leq|\widetilde{\eta}-\eta| \sum_{k=0}^{\infty} L^{k}\left[\begin{array}{c}
k \alpha+\alpha \\
n
\end{array}\right]=|\widetilde{\eta}-\eta| F_{\alpha, \alpha}(L, n) .
\end{aligned}
$$

Then the solutions of (3.1) rely on the initial value continuously.

\section{Competing interests}

The authors declare that they have no competing interests.

\section{Authors' contributions}

RX studied the generalized discrete fractional Gronwall inequalities and completed the corresponding proof. YZ obtained the results of Section 3: Applications. All authors read and approved the final manuscript.

\section{Author details}

'Department of Mathematics, Qufu Normal University, Qufu, Shandong 273165, People's Republic of China. ${ }^{2}$ Basic Department of Shandong Water Polytechnic, Rizhao, Shandong 276800, People's Republic of China.

\section{Acknowledgements}

The authors are very grateful to the anonymous referees for their valuable suggestions and comments, which helped to improve the quality of the paper. The author RX is supported by the National Science Foundation of China (11171178 and 11271225).

\section{Received: 6 May 2015 Accepted: 16 July 2015 Published online: 04 August 2015}

\section{References}

1. Miller, KS, Ross, B: An Introduction to the Fractional Calculus and Fractional Differential Equations. Wiley, New York (1993)

2. Samko, SG, Kilbas, AA, Maitchev, OI: Fractional Integrals and Derivatives. Theory and Applications. Nauka i Technika, Minsk (1987)

3. Miller, KS, Ross, B: Fractional difference calculus. In: Univalent Functions, Fractional Calculus, and Their Applications, pp. 139-152. Ellis Horwood, Chichester (1989)

4. Atici, FM, Sengul, SS: Modeling with discrete fractional equations. J. Math. Anal. Appl. 369, 1-9 (2010)

5. Abdeljawad, T, Baleanu, D: Caputo $q$-fractional initial value problems and a $q$-analogue Mittag-Leffler function. Commun. Nonlinear Sci. Numer. Simul. 16(12), 4682-4688 (2011). doi:10.1016/j.cnsns.2011.01.026

6. Abdeljawad, T, Baleanu, D: Fractional differences and integration by parts. J. Comput. Anal. Appl. 13(3), 574-582 (2011)

7. Atici, FM, Eloe, PW: A transform method in discrete fractional calculus. Int. J. Difference Equ. 2(2), 165-176 (2007)

8. Atici, FM, Eloe, PW: Initial value problems in discrete fractional calculus. Proc. Am. Math. Soc. 137(3), 981-989 (2009)

9. Atici, FM, Sengul, S: Modeling with fractional difference equations. J. Math. Anal. Appl. 369(1), 1-9 (2010)

10. Atici, FM, Eloe, PW: Two-point boundary value problems for finite fractional difference equations. J. Differ. Equ. Appl. $17,445-456(2011)$

11. Bastos, NRO, Ferreira, RAC, Torres, DFM: Necessary optimality conditions for fractional difference problems of the calculus of variations. Discrete Contin. Dyn. Syst. 29(2), 417-437 (2011)

12. Cheng, J, Chu, Y: On the fractional difference equations of order (2, q). Abstr. Appl. Anal. 2011, Article ID 497259 (2011). doi:10.1155/2011/497259

13. Cheng, J: The solution of fractional difference equations of order (k, q). Acta Math. Appl. Sin. 34(2), 313-330 (2011)

14. Cheng, J, Chu, Y: Fractional difference equations with real variable. Abstr. Appl. Anal. 2012, Article ID 918529 (2012)

15. Goodrich, CS: Solutions to a discrete right-focal fractional boundary value problem. Int. J. Difference Equ. 5, 195-216 (2010)

16. Goodrich, CS: Continuity of solutions to discrete fractional initial value problems. Comput. Math. Appl. 59(11) 3489-3499 (2010) 
17. Jarad, F, Abdeljawad, T, Baleanu, D: On the stability of some discrete fractional nonautonomous systems. Abstr. Appl. Anal. 2012, Article ID 476581 (2012)

18. Hirota, R: Lectures on Difference Equations. Science-sha, Tokyo (2000) (in Japanese)

19. Nagai, A: An integrable mapping with fractional difference. J. Phys. Soc. Jpn. 72, 2181-2183 (2003)

20. Deekshitulu, GVSR, Jagan Mohan, J: Fractional difference inequalities. Commun. Appl. Anal. 14, $89-97$ (2010)

21. Deekshitulu, GVSR, Jagan Mohan, J: Fractional difference inequalities of Bihari type. Commun. Appl. Anal. 14, 343-354 (2010)

22. Deekshitulu, GVSR, Jagan Mohan, J: Fractional difference inequalities of Opial type and initial value problem. Fract. Differ. Calc. 2, 73-85 (2012)

23. Deekshitulu, GVSR, Jagan Mohan, J: Some new fractional difference inequalities. In: ICMMSC 2012. CCIS, vol. 283, pp. 403-412. Springer, Berlin (2012)

24. Deekshitulu, GVSR, Jagan Mohan, J, Anand, PVS: Fractional difference inequalities of Volterra type. Int. J. Pure Appl. Math. 70, 137-149 (2011)

25. Deekshitulu, GVSR, Jagan Mohan, J: Fractional difference inequalities of Gronwall-Bellman type. Acta Comment. Univ. Tartu Math. 17(1), 19-30 (2013) www.math.ut.ee/acta/

26. Atici, FM, Eloe, PW: Discrete fractional calculus with the nabla operator. Electron. J. Qual. Theory Differ. Equ. Special Edition I, 3 (2009)

27. Atici, FM, Eloe, PW: Gronwall's inequality on discrete fractional calculus. Comput. Math. Appl. 64, 3193-3200 (2012)

28. Ferreira, RAC: A discrete fractional Gronwall inequality. Proc. Am. Math. Soc. 140, 1605-1612 (2012)

29. Cheng, J: The Theory of Fractional Difference Equations. Xiamen University Press, Xiamen (2011) (in Chinese)

30. Zhang, Y, Huang, J, Xu, X, Yang, Y: A class of discrete fractional Gronwall inequalities. Math. Pract. Theory 6, 222-225 (2014)

\section{Submit your manuscript to a SpringerOpen ${ }^{\circ}$ journal and benefit from:}

- Convenient online submission

- Rigorous peer review

- Immediate publication on acceptance

- Open access: articles freely available online

- High visibility within the field

- Retaining the copyright to your article 